\title{
Preparation of ultrafine uniform electrospun polyimide nanofiber
}

\author{
Satoshi Fukushima, Yuki Karube and Hiroyoshi Kawakami
}

This paper reports the preparation of bead-free ultrafine uniform nanofibers with a narrow fiber diameter distribution from fluorinated polyimide by electrospinning. Increasing the electrical conductivity of polymer solutions by the addition of salts and decreasing the discharge rate of water from the polymer by decreasing the humidity in the surrounding air decreases the diameter of the electrospun nanofiber. Ultrafine uniform nanofibers were achieved using these new salt and humidity conditions, and we successfully prepared bead-free ultrafine uniform nanofibers with a narrow range of nanoscale diameters ( $33 \pm \mathbf{n m}$ ). Polymer Journal (2010) 42, 514-518; doi:10.1038/pj.2010.33; published online 28 April 2010

Keywords: electrospinning; fluorinated polyimide; humidity; nanofiber; salt

\section{INTRODUCTION}

Nanoscale fibers prepared by the electrospinning method have received a great deal of attention, as electrospinning is capable of producing fibers with diameters in the nanometer range. ${ }^{1-4}$ Electrospun nanofibers possess many unique properties including a large specific surface area and superior mechanical properties, and they have potential use as nanoscale building blocks. Nanofibers have been successfully prepared for many applications such as filters, optical and chemical sensors, catalyst systems, scaffolds for tissue regeneration and immobilized enzymes. ${ }^{5-10}$ However, the applications are currently somewhat limited by the fact that most electrospun nanofibers are in the form of nonwoven mats composed of disordered nanofibers. Aligned nanofibers can be used in a variety of electrical, optical, mechanical and biomedical applications.

Recently, we reported the synthesis of novel nanofibrous fluorinated polyimide membranes using an electrospinning method on a specially designed collector composed of conductive aluminum plates and glass insulator materials that can be removed from the apparatus. ${ }^{11}$ The electrospun nanofibers were deposited across the plates and uniaxially aligned to the collector. In addition, multilayer stacked nanofibrous membranes consisting of three-dimensionally ordered nanopores were produced, and the water flux properties of the nanofibrous membranes were measured. The distance between the uniaxially aligned nanofibers decreased with deposition time, and the collector resulted in square nanopores with a regular diameter of approximately $200 \mathrm{~nm}$. The nanopores prepared by the uniaxially aligned nanofibers had a significantly more regular structure than that formed by nonwoven nanofibers.

The nanopore size or the distance between the uniaxially aligned nanofibers strongly depended on the nanofiber diameter. Many papers report a uniform nanofiber without beads with a diameter of approximately $100 \mathrm{~nm}$. We prepared a uniform nanofiber with a diameter of less than $50 \mathrm{~nm}$ because such nanofibrous membranes could enhance both the flux and selectivity of size-exclusion membranes, and are also useful for many applications such as catalysis, as well as in electronic and photonic devices and tissue scaffolds. ${ }^{12-14}$

To fabricate the desired ultrafine uniform nanofiber, we focused on the effects of salt additives in the polymer solution and humidity conditions when the polymer solution is electrospun. The electrical conductivity of the solution is known to have an important role in the formation of nonbeaded nanofibers. ${ }^{15}$ It has been reported for many water-soluble polymers that increasing the electrical conductivity of polymer solutions by the addition of inorganic salts can decrease the diameter of electrospun nanofibers. ${ }^{16-18}$ Lee et al. ${ }^{17}$ reported that a uniform electrolyte polymer nanofiber with a diameter of approximately $70 \mathrm{~nm}$ was prepared by the addition of an ionic salt at $0.01 \mathrm{~mol} \% .^{19}$

On the other hand, Choi et al. ${ }^{20}$ investigated the effect of organosoluble salts on the nanofibrous structure of electrospun poly(3hydroxybutyrate-co-3-hydroxyvalerate) ${ }^{20}$ When small amounts of benzyl trialkylammonium chlorides as the organosoluble salt were added to the poly(3-hydroxybutyrate-co-3-hydroxyvalerate) solution, the average diameter decreased to $1.0 \mu \mathrm{m}$ and the fibers were straight. However, there is no report of an organosoluble polymer nanofiber with a diameter of less than $100 \mathrm{~nm}$ produced by the addition of organosoluble salts.

In addition, we considered that the humidity at electrospinning may influence the morphology or diameter of nanofibers, because if the humidity in the surrounding air is increased, the dischargerate of water from the polymer is rapidly increased. ${ }^{21}$ However, few papers have considered the correlation between nanofiber diameter and humidity. 
This study describes an electrospinning method with new salt and humidity conditions to prepare bead-free ultrafine uniform nanofibers with a narrow fiber-diameter distribution.

\section{EXPERIMENTAL PROCEDURE}

\section{Materials}

2,2'-Bis(3,4-dicarboxyphenyl)hexafluoropropane dianhydride (6FDA) and 2,2'bis(4-aminophenyl)hexafluoropropane (6FAP) were purchased from the Central Glass (Saitama, Japan). 6FDA was purified by sublimation before use. 6FAP was recrystallized twice from an ethanol solution before use. Tetrabutyl ammonium bromide (TBAB), a salt soluble in organic solvents, was purchased from KANTO CHEMICAL (Tokyo, Japan) and used without purification.

\section{Synthesis and characteristics of 6FDA-6FAP}

Polyimide 6FDA-6FAP was synthesized by chemical imidization of poly(amic acid) precursors as reported in literature. ${ }^{20-22}$ Figure 1 shows the structures of 6FDA-6FAP. The molecular weights $\left(M_{\mathrm{w}}\right.$ and $\left.M_{\mathrm{n}}\right)$ of polyimides were determined by gel permeation chromatography (detector: Jasco 830-RI monitor, Jasco, Tokyo, Japan), with tetrahydrofuran as the solvent. A flow rate of $1.0 \mathrm{ml} \mathrm{min}^{-1}$ was used, and the polyimide was dissolved in tetrahydrofuran at a concentration of $0.005 \mathrm{wt} \%$. Molecular weights were estimated by comparing the retention times on a column (Shodex KF-805L, Shodex, Tokyo, Japan) with those of standard polystyrene.

\section{Nanofiber preparation}

Figure 2 shows the experimental setup used for the preparation of nanofibers. Nanofibers were fabricated using an electrospinning apparatus (Fuence, ES1000, Tokyo, Japan). In this study, a $5 \mathrm{~cm} \times 5 \mathrm{~cm}$ aluminum plate was used. Relative humidity during electrospinning was controlled by the infill nitrogen gas.

6FDA-6FAP was dissolved in dimethyl formamide. The distance between the spinneret and the grounded plate varied from 20 to $10 \mathrm{~cm}$. We performed a detailed investigation of electrospinning parameters such as polymer concentration, the injection speed through the needle and the voltage between the syringe and the collector to prepare the ultrafine nanofiber. The effects of the addition of salt to the polymer solution and the variation of humidity conditions during electrospinning on the nanofiber diameter were also examined. Finally, vacuum drying was carried out at $150^{\circ} \mathrm{C}$ for $10 \mathrm{~h}$ to remove the residual solvent from the fabricated nanofibers.<smiles>CN1C(=O)c2ccc(C(C)(C)c3ccc4c(c3)C(=O)N(c3ccc(C(C)(C)C)cc3)C4=O)cc2C1=O</smiles>

Figure 1 Chemical structure of 6FDA-6FAP.

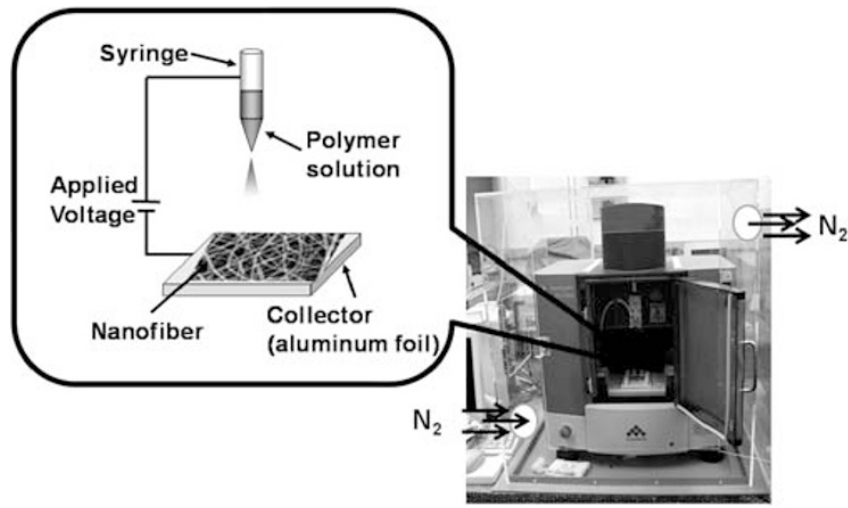

Figure 2 Electrospinning apparatus.
Nanofiber morphology, including diameter, was determined using a scanning electron microscope (JXP-6100P; JEOL, Tokyo, Japan). To determine the diameter, electrospun nanofibers $(3 \mathrm{~cm} \times 3 \mathrm{~cm})$ were fixed on a flat sample holder. The nanofibers were coated with gold for $120 \mathrm{~s}$ at a current of $10 \mathrm{~mA}$ under a vacuum of $10 \mathrm{~Pa}$. At least five pictures were used to determine the diameter.

\section{RESULTS AND DISCUSSION}

Polyimide 6FDA-6FAP was synthesized by chemical imidization by treatment with a mixture of acetic anhydride and triethylamine. The degree of imidization of the polyimide was measured by ${ }^{1} \mathrm{H}-\mathrm{NMR}$. The synthesized 6FDA-6FAP was soluble in dimethyl formamide, DMAc, dimethylsulfoxide and NMP, and showed high molecular weight $\left(5.5 \times 10^{5}\right)$ with a narrow polydispersity index (1.8).

It has been reported that the shape of the initiating droplet through the spinneret can be changed by the conditions of the electrospinning apparatus such as the applied voltage and feed rate of the polymer solution and the distance between the spinneret and the grounded collector plate. We confirm the influence of these conditions on the diameter of electrospun 6FDA-6FAP nanofiber. The nonwoven 6FDA-6FAP nanofibers were electrospun on a grounded aluminum plate. The distance between the spinneret and the grounded plate was $10 \mathrm{~cm}$, and the concentration of the polymer solution was $10 \mathrm{wt} \%$.

It was previously reported that the applied voltage and feed rate do not have significant effects on the diameter of electrospun nanofibers. $^{22,23}$ Figure 3 clearly shows that the diameter of the nanofiber depended on the applied voltage and feed rate, although the effect was not dramatic. According to previous studies, a higher voltage was reported to induce not only a larger diameter but also a smaller diameter, because the higher voltage has different effects on different polymers or under different conditions. In this study, we consider that applied voltage affects jet elongation, as elongation is controlled by electrical force; that is, the nanofiber was elongated at high voltage by strong electrostatic repulsion so that the diameter of the nanofiber was decreased. The diameter also decreased with a decrease in the feed rate of the polymer solution.

Significant morphological changes were observed with changes in the concentration or viscosity of the polymer solution, which means that the concentration or viscosity of the polymer solution was one of the most effective variables for controlling nanofiber morphology. Therefore, we studied the effect of the molecular weight of 6FDA$6 \mathrm{FAP}$ on the diameter of the electrospun nanofiber using 6FDA-6FAP, with molecular weights of $1.2 \times 10^{5}, 2.2 \times 10^{5}, 4.2 \times 10^{5}$ and $5.5 \times 10^{5}$.
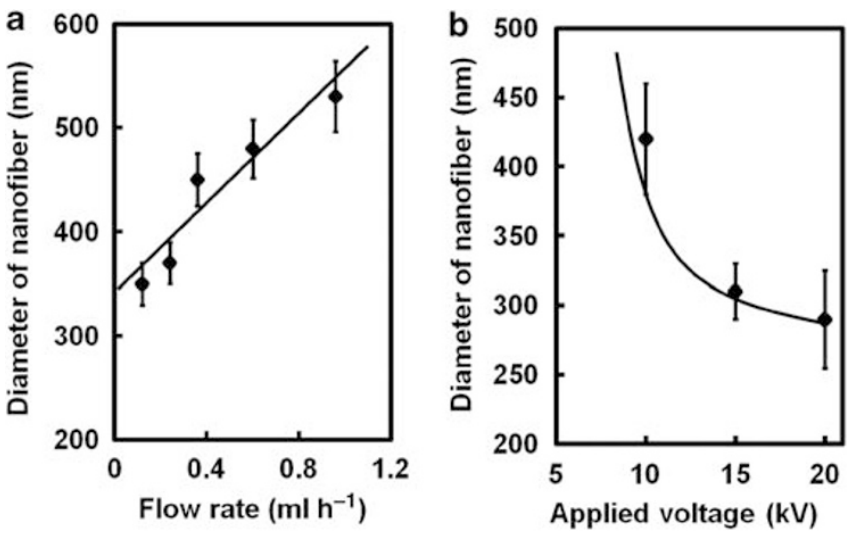

Figure 3 Effects of flow rate and applied voltage on nanofiber diameter at $32{ }^{\circ} \mathrm{C}$. 
Figure 4 presents scanning electron microscope images of the 6FDA6FAP nanofibers electrospun on a grounded aluminum plate, demonstrating that it is impossible to fabricate a continuous nanofiber at a concentration of 6FDA-6FAP in dimethyl formamide below 18, 13, 9 and $9 \mathrm{wt} \%$ for molecular weights of $1.2 \times 10^{5}, 2.2 \times 10^{5}, 4.2 \times 10^{5}$ and $5.5 \times 10^{5}$, respectively, and that the nanofibers were composed of a mixture of fibers and beads. On the other hand, when electrospun at $19,14,10$ and $10 \mathrm{wt} \%$ concentrations, uniform nanofibers were produced, as the polymer solution was sufficiently viscous. ${ }^{24,25}$

Figure 5 shows the effects of the molecular weight of the polymer and the viscosity of the polymer solution on the nanofiber diameter. Polymers with molecular weights of $1.2 \times 10^{5}, 2.2 \times 10^{5}, 4.2 \times 10^{5}$ and $5.5 \times 10^{5}$ were prepared at concentrations of $19,14,12$ and $10 \mathrm{wt} \%$, respectively. The molecular weight of the polymer is related to the number of entanglements of the polymer chains in solution, and thus to the viscosity of the polymer solution. The nanofiber diameter decreased with increasing molecular weight of 6FDA-6FAP because of

a $\operatorname{Mw}\left(\times 10^{5}\right)$

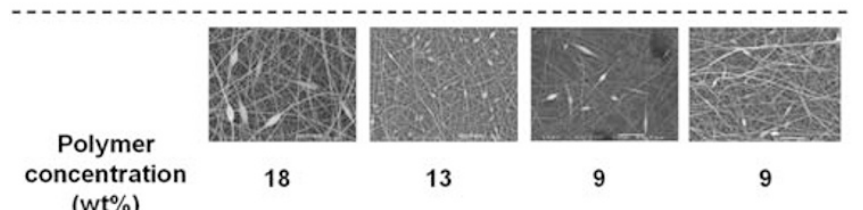

b

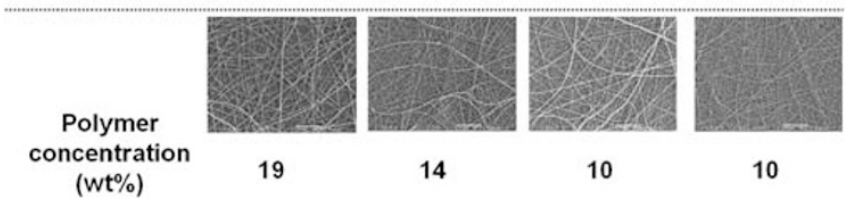

Figure 4 Scanning electron microscope images of 6FDA-6FAP nanofibers (a) with beads and (b) without beads. the increased solution viscosity. Therefore, the molecular weight of the polymer had an important role in determining the nanofiber diameter.

After optimizing the electrospinning parameters such as the applied voltage, the feed rate of the 6FDA-6FAP solution and the molecular weight of 6FDA-6PAF, the mean diameter of the continuous nanofibers without beads was $199 \pm 27 \mathrm{~nm}(n=25)$. These results indicate that it is very difficult to prepare the 6FDA-6FAP nanofiber with a diameter less than $100 \mathrm{~nm}$ using the parameters discussed above. Therefore, we focused on the effects of salt additives and humidity to prepare an ultrafine uniform electrospun nanofiber.

In general, the morphology of electrospun nanofibers is considered to be primarily affected by the concentration, viscosity and electrical conductivity of the polymer solution. The organosoluble salt TBAB was added to increase the conductivity of the 6FDA-6FAP solution. Figure 6 shows the diameters of nanofibers electrospun with polymer solutions containing TBAB. Addition of a small amount of TBAB to the polymer solution decreased the mean diameter of the nanofibers by a factor of two. In addition, the nanofibers were uniform and continuous, without beads. On the other hand, the viscosity of the polymer solution decreased slightly with the addition of TBAB. In general, the electrical conductivity of a polymer solution reflects the charge density of a jet so that the elongation of the jet occurs because of an enhanced electrical force; that is, the addition of salt enhanced the elongation of the jet, as well as the conductivity. Therefore, electrospun nanofibers were produced because of the formation of a stable jet resulting from the sufficient transfer of charges into the jet, and the fiber diameters were smaller because of the elongation of the jet.

It is well known that polymer charging is affected by environmental conditions. Adsorbed water molecules provided by the surrounding air can contribute to the mobility of charge carriers and promote their diffusion. Therefore, we considered that the humidity during electrospinning could be an important factor determining the diameter of electrospun nanofibers. Figure 7 shows the effect of humidity at electrospinning on the nanofiber diameter. The diameter strongly depended on humidity and dramatically decreased with decreasing

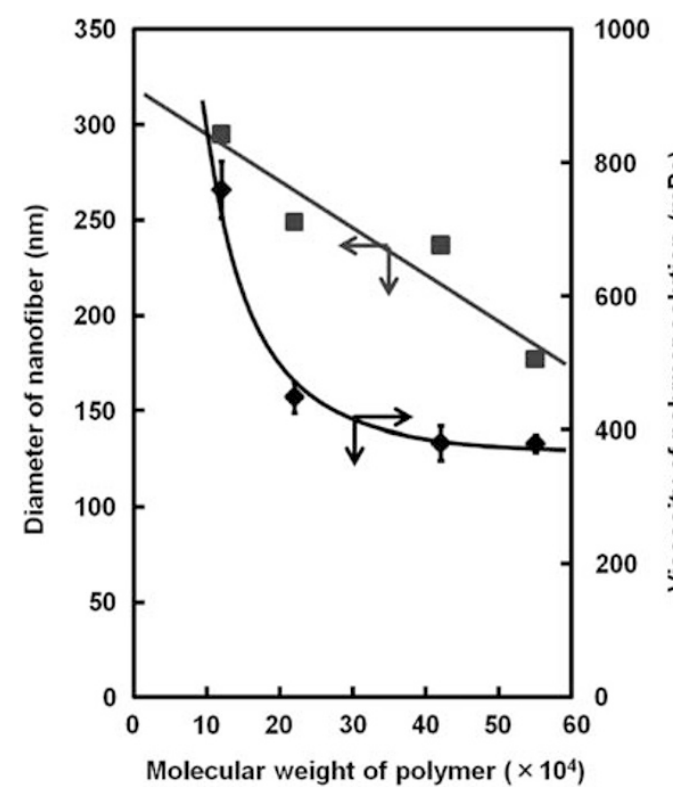

Figure 5 Effects of polymer molecular weight and polymer solution viscosity on nanofiber diameter at $32^{\circ} \mathrm{C}$.

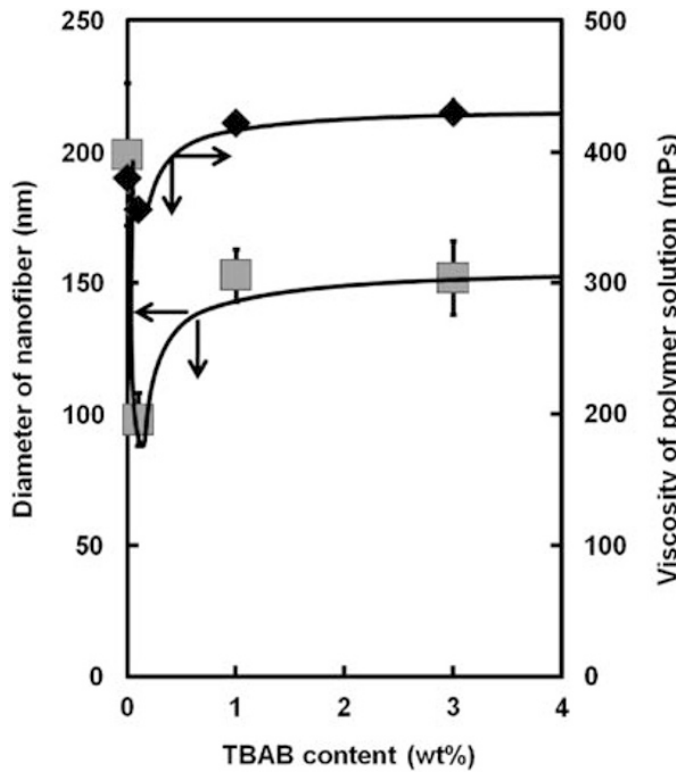

Figure 6 Effects of TBAB content and polymer solution viscosity on nanofiber diameter at $32^{\circ} \mathrm{C}$. 


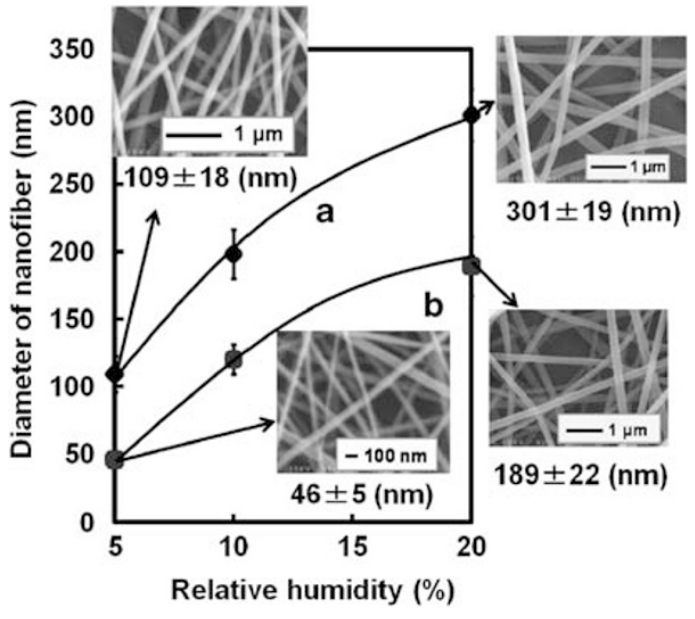

Figure 7 Effects of TBAB and relative humidity on nanofiber diameter at $32{ }^{\circ} \mathrm{C}$ with (a) 0 wt $\%$ TBAB and (b) 0.1 wt $\%$ TBAB.

Distance between nozzle and collector $(\mathrm{cm})$

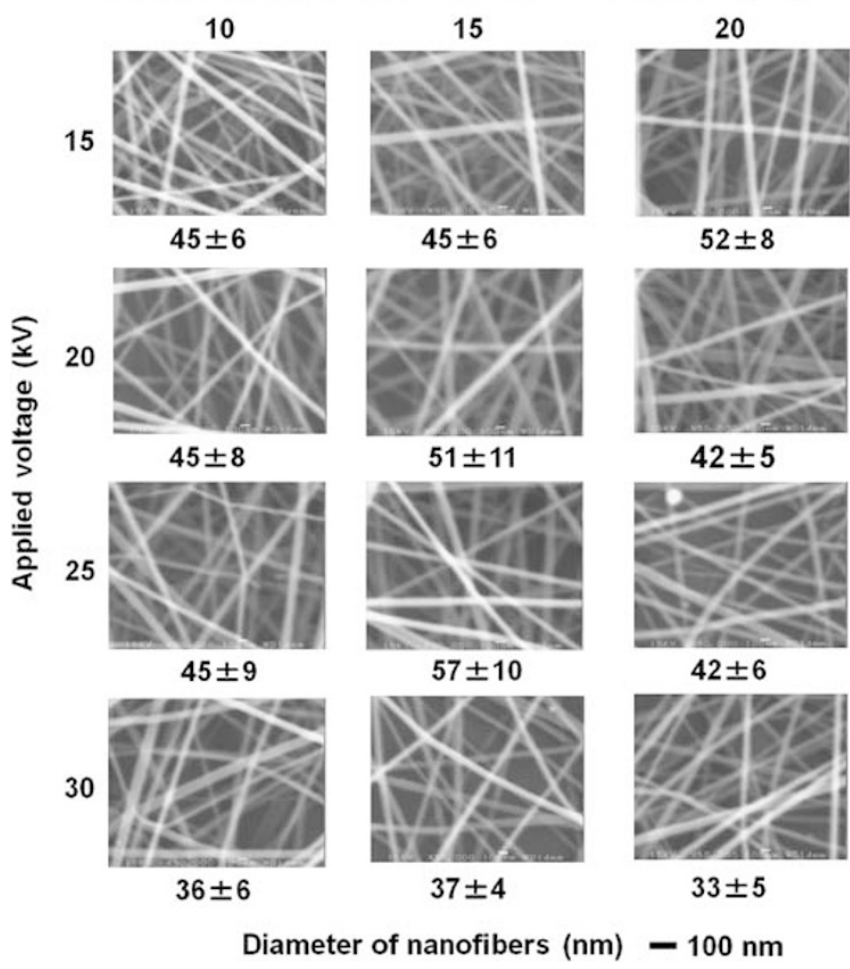

Figure 8 Effects of applied voltage and distance between the nozzle and the collector on the preparation of nanofibers at $32{ }^{\circ} \mathrm{C}$.

humidity. The mean diameter of electrospun nanofibers prepared in a polymer solution containing $\mathrm{TBAB}$ at $5 \%$ relative humidity was $46 \pm 5 \mathrm{~nm} \quad(n=25)$, which was approximately one-fourth of the diameter at $20 \%$ relative humidity. Reducing humidity enabled the preparation of a uniform nanofiber with a diameter less than $50 \mathrm{~nm}$. We believe that the water molecules provided by the surrounding air at electrospinning have a significant influence on the diameter of 6FDA-6FAP nanofibers because the water molecules are responsible for the charge transport that involves the discharging of the 6FDA6FAP solution or the 6FDA-6FAP nanofiber surface. Therefore, greater humidity in the surrounding air increased the discharge rate, and the conductivity of the polymer solution or the polymer nanofiber surface decreased. As a result, humidity reduced the jet length and increased the nanofiber diameter.

Figure 8 shows the scanning electron microscope images of nanofibers prepared with these optimized electrospinning conditions, demonstrating a successful preparation of a uniform, nonbeaded nanofiber with a diameter of less than $40 \mathrm{~nm}$.

\section{CONCLUSIONS}

In this study we investigated the effects of electrospinning parameters such as applied voltage, feed rate, viscosity of the polymer solution, salt addition and humidity on the diameter and uniformity of electrospun nanofibers, and optimization of these parameters enabled the preparation of bead-free ultrafine uniform nanofibers. Fluorinated polyimide uniform nonbeaded nanofibers were prepared with a diameter of less than $40 \mathrm{~nm}$.

This study has served two important purposes. First, we demonstrated the influence of salt and humidity during electrospinning on the size and uniformity of nanofibers. Second, optimization of these parameters enabled the production of a new class of nanofibers that enhances the flux and selectivity of size-exclusion membranes, with potential applications in catalysis, electronic and photonic devices and tissue scaffolds.

\section{ACKNOWLEDGEMENTS}

This work was partially supported by a grant from the New Energy and Industrial Technology Development Organization.

1 Nasir, M., Matsumoto, H., Minagawa, M., Tanioka, A., Danno, T. \& Horibe, H. Preparation of PVDF/PMMA blend nanofibers by electrospray deposition: effects of blending ratio and humidity. Pol. J. 41, 402-406 (2009).

2 Vetcher, A. A., Gearheart, R. \& Morozov, V. N. Correlation of morphology of electrospun fibers with rheology of linear polyacrylamide solution. Pol. J. 39, 878-881 (2007).

$3 \mathrm{Ma}$, Z., Kotaki, M. \& Ramakrishna, S. Electrospun cellulose nanofiber as affinity membrane. J. Membr. Sci. 265, 115-123 (2005).

4 Yang, F., Murugan, R., Wang, S. \& Ramakrishn, S. Electrospinning of nano/micro scale poly(L-lactic acid) aligned fibers and their potential in neural tissue engineering. Biomaterials 26, 2603-2610 (2005).

5 Gopal, R., Kaur, S., Ma, Z., Chan, C., Ramakrishna, S. \& Matsuura, T. Electrospun nanofibrous filtration membrane. J. Membr. Sci. 281, 581-586 (2006).

6 Sawicka, K., Gouma, P. \& Simon, S. Electrospun biocomposite nanofibers for urea biosensing. Sens. Actuators B. 108, 585-588 (2005).

7 Chen, L., Bromberg, L., Hatton, T. A. \& Rutledge, G. C. Catalytic hydrolysis of p-nitrophenyl acetate by electrospun polyacrylamidoxime nanofibers. Polymer 48, 4675-4682 (2007).

8 Chua, K. N., Lim, W. S., Zhang, P., Lu, H., Wen, J., Ramakrishna, S., Leong, K. W. \& Mao, H. Q. Stable immobilization of rat hepatocyte spheroids on galactosylated nanofiber scaffold. Biomaterials 26, 2537-2547 (2005).

9 Badami, A. S., Kreke, M. R., Thompson, M. S., Riffle, J. S. \& Goldstein, A. S. Effect of fiber diameter on spreading, proliferation, and differentiation of osteoblastic cells on electrospun poly(lactic acid) substrates. Biomaterials 27, 596-606 (2006).

$10 \mathrm{Wu}$, L., Yuan, X. \& Sheng, J. Immobilization of cellulase in nanofibrous PVA membranes by electrospinning. J. Membr. Sci. 250, 167-173 (2005)

11 Karube, Y. \& Kawakami, H. Fabrication of well-aligned electrospun nanofibrous membrane based on fluorinated polyimide. Polym. Adv. Tech. (e-pub ahead of print 16 July 2009; doi:10.1002/pat.1511).

$12 \mathrm{Li}$, D., Wang, Y. \& Xia, Y. Electrospinning of polymeric and ceramic nanofibers as uniaxially aligned arrays. Nano Lett. 3, 1167-1171 (2003).

13 Li, D., Ouyang, G., McCann, J. T. \& Xia, Y. Collecting electrospun nanofibers with patterned electrodes. Nano Lett. 5, 913-916 (2005).

14 Boland, E. D., Pawlowski, K. J., Barnes, C. P., Simpson, D. G., Wnek, G. E. \& Bowlin, G. $\mathrm{L}$. Electrospinning of bioresorbable polymers for tissue engineering scaffolds. Polymeric Nanofibers 14, 188-204 (2006).

15 Tan, S- H., Inai, R., Kotaki, M. \& Ramakrishna, S. Systematic parameter study for ultrafine fabrication via electrospinning process. Polymer 46, 6128-6134 (2005). 
16 Chronakis, I. S., Grapenson, S. \& Jakob, A. Conductive polypyrrole nanofibers via electrospinning: electrical and morphological properties. Polymer 47, 1597-1603 (2006).

17 Lee, C. K., Kim, S. I. \& Kim, S. J. The influence of added ionic salt on nanofiber uniformity for electrospinning of electrolyte polymer. Synthetic Metals. 154, 209-212 (2005).

18 Zhang, H., Song, H., Dong, B., Han, L., Pan, G., Bai, X., Fan, L., Lu, S., Zhao, H. \& Wang, F. Electrospinning preparation and luminescence properties of Europium complex/polymer composite fibers. J. Phys. Chem. C. 112, 9155-9162 (2008).

19 Lin, K., Chu, K.- N., Christopherson, G. T., Lim, S. \& Mao, H.- Q. Reducing electrospun nanofiber diameter and variability using cationic amphiphiles. Polymer 48, 63846394 (2007).

20 Choi, J. S., Lee, S. W., Jeong, L., Bae, S- H., Min, B. C., Youk, J. H. \& Park, W. H. Effect of organosoluble salts on the nanofibrous structure of electrospun poly(3-hydroxybutyrate-co-3-hydroxyvalerate). Int. J. Biol. Macro. 34, 249-256 (2004).
21 Kawakami, H., Anzai, J. \& Nagaoka, S. Gas transport properties of soluble aromatic polyimides with sulfone diamine moieties. J. Appl. Polym. Sci. 57, 789795 (1995).

22 Kawakami, H., Mikawa, M. \& Nagaoka, S. Gas transport properties in thermally cured aromatic polyimide membranes. J. Membr. Sci. 118, 223-230 (1996).

23 Kawakami, H., Mikawa, M., Takagi, J. \& Nagaoka, S. Gas transfer and blood compatibility of fluorinated polyimide membranes. J. Biomater. Sci. Polym. Ed. 7, 1029-1038 (1996).

24 Gupta, P., Elkins, C., Long, T. E. \& Wilkes, G. L. Electrospinning of linear homopolymers of poly(methyl methacrylate): exploring relationships between fiber formation, viscosity, molecular weight and concentration in a good solvent. Polymer 46, 4799-4810 (2005).

25 Thompson, C. J., Chase, G. G., Yarin, A. L. \& Reneker, D. H. Effects of parameters on nanofiber diameter determined from electrospinning model. Polymer 48, 6913-6922 (2007). 